\title{
Scalar concentration measurements in liquid-phase flows with pulsed lasers
}

\author{
Jerry W. Shan, Daniel B. Lang, Paul E. Dimotakis
}

Exp Fluids (2004) 36:268-273

Due to an unfortunate error some of the corrections marked in the proof have not been carried out accurately. We regret this and herewith publish the correct information.

1. Last sentence of section 2 Optics and instrumentation:

For instance, a light sheet formed by spreading a $250 \mathrm{~mJ}, 6 \mathrm{~ns}$ laser pulse to a width of $1.0 \mathrm{~m}$ and a thickness of $0.2 \mathrm{~mm}$, has an instantaneous power density of the order $2 \times 10^{11} \mathrm{~W} / \mathrm{m}^{2}$.

2. Correct Eqs. 6 and 17:

$$
\begin{aligned}
& \frac{\partial}{\partial I_{\mathrm{L}}} I_{\mathrm{F}}\left(I_{L} ; C\right)=\left[a\left(I_{L} ; C\right)+\frac{\partial}{\partial I_{\mathrm{L}}} a\left(I_{L} ; C\right) I_{L}\right] C . \\
& \frac{C(x, y, t)}{C_{\text {ref }}}=\frac{I_{\mathrm{F}}(x, y, t)-\left\langle I_{\mathrm{F}, \text { back }}(x, y)\right\rangle}{\left\langle I_{\mathrm{F}, \text { ref }}(x, y)\right\rangle-\left\langle I_{\mathrm{F}, \text { back }}(x, y)\right\rangle} .
\end{aligned}
$$

3. The third-last sentence of section $\mathbf{5}$ Conclusion:

Despite this fluorescence saturation, the fluorescence intensity increases linearly with dye concentration for $0.1 \times 10^{-6} \leq C \leq 1.0 \times 10^{-6} \mathrm{M}$.

E-mail: jshan@jove.rutgers.edu 